\title{
Activational effects of estradiol and dihydrotestosterone on social recognition and the arginine-vasopressin immunoreactive system in male mice lacking a functional aromatase gene
}

\author{
S. Pierman ${ }^{\mathrm{a}, 1}$, M. Sica ${ }^{\mathrm{b}, 1}$, F. Allieri ${ }^{\mathrm{b}}$, C. Viglietti-Panzica ${ }^{\mathrm{c}}$, G.C. Panzica ${ }^{\mathrm{c}}$, J. Bakker ${ }^{\mathrm{a}, *}$ \\ ${ }^{a}$ Centre for Cellular and Molecular Neurobiology, University of Liège, Avenue de l'hopital 1 (B36), 4000 Liège, Belgium \\ ${ }^{\mathrm{b}}$ Laboratory of Neuroendocrinology, Rita Levi Montalcini Centre for Brain Repair, Department of Anatomy, Pharmacology and Forensic Medicine, \\ University of Torino, Torino, Italy \\ ${ }^{\mathrm{c}}$ Laboratory of Neuroendocrinology, Department of Anatomy, Pharmacology and Forensic Medicine, University of Torino and Instituto Nazionale di Neuroscienze, \\ Torino, Italy
}

Received 31 October 2007; revised 31 January 2008; accepted 1 February 2008

Available online 15 February 2008

\begin{abstract}
In rodents, parts of the arginine-vasopressin (AVP) neuronal system are sexually dimorphic with males having more AVP-immunoreactive cells/ fibers than females. This neuropeptide neuronal system is highly sensitive to steroids and has been proposed to play an important role in the processing of olfactory cues critical to the establishment of a social memory. We demonstrate here that gonadally intact male aromatase knockout (ArKO) mice, which cannot aromatize androgens into estrogens due to a targeted mutation in the aromatase gene, showed severe deficits in social recognition as well as a reduced AVP-immunoreactivity in several brain regions. To determine whether this reduction is due to a lack of organizational or activational effects of estrogens, we assessed social recognition abilities and AVP-immunoreactivity in male ArKO and wild-type (WT) mice when treated with estradiol benzoate (EB) in association with dihydrotestosterone propionate (DHTP) in adulthood. Adult treatment with EB and DHTP restored social recognition abilities in castrated ArKO males since they showed normal female-oriented ultrasonic vocalizations and were able to recognize an unfamiliar female using a habituation-dishabituation paradigm. Furthermore, adult treatment also restored AVP-immunoreactivity in the lateral septum of ArKO males to levels observed in intact WT males. These results suggest that social recognition in adulthood and stimulation of AVP expression in the adult mouse forebrain depend predominantly on the estrogenic metabolite of testosterone. Furthermore, our results are in line with the idea that the organization of the AVP system may depend on androgen or sex chromosomes rather than estrogens.
\end{abstract}

(C) 2008 Elsevier Inc. All rights reserved.

Keywords: Testosterone; Aromatase; Estradiol; Dihydrotestosterone; Vasopressin; Bed nucleus of stria terminalis; Lateral septum; Hypothalamus

\section{Introduction}

The ability to recognize a conspecific is fundamental for all social relationships, including parent-offspring recognition, mate recognition, and dominant-subordinate hierarchies. Rodent species, including mice, use primarily olfactory cues to recognize conspecifics (Brown, 1979). The neuropeptide arginine-vasopressin (AVP) has been proposed to play an important

\footnotetext{
* Corresponding author. Fax: +32 43665971. E-mail address: jbakker@ulg.ac.be (J. Bakker).

${ }^{1}$ These authors contributed equally to the presented work.
}

role in the processing of olfactory cues critical to the establishment of a social memory (reviewed in Bielsky and Young, 2004). AVP is produced in discrete regions of the brain and is released centrally in the brain as well as peripherally, where it acts as an anti-diuretic hormone. Centrally, AVP projections to the lateral septum are primarily coming from the bed nucleus of the stria terminalis (BNST) and the medial amygdala (Me) in the rat (De Vries and Buijs, 1983).

In rats and mice, as well as in several non-mammalian species, parts of the arginine-vasopressin (AVP) neuronal system are sexually dimorphic and sensitive to sex steroids (Bakker et al., 2006; De Vries and Boyle, 1998; De Vries and Miller, 1998; De Vries and Panzica, 2006). For instance, the BNST and the Me contain more AVP-immunoreactive (-ir) in males than in 
females. Furthermore, the AVP-ir projections from these nuclei to the lateral septum (LS) are denser in males than in females. These sex differences do not depend on differences in circulating hormone levels in adulthood, since they persist in males and females which are treated with similar levels of testosterone (De Vries and Al Shamma, 1990; De Vries et al., 1994a,b) or estradiol (Bakker et al., 2006), but are primarily due to differences in hormone levels during development. Treatment of newborn female rats with testosterone induces a male-typical AVP distribution (Han and De Vries, 2003). These masculinizing effects of testosterone on the AVP system may be mediated predominantly by its aromatized metabolite since neonatal treatment of newborn female rats with estradiol benzoate (EB) or with EB and DHTP increased the number of AVP mRNA containing cells in the BNST more than neonatal treatment with DHTP alone (Han and De Vries, 2003). By contrast, in mice, these sex differences may not depend on the presence of estrogens during early development since female alpha-fetoprotein knockout (AFP-KO) mice which are exposed to excessive levels of estrogens prenatally since they lack the protective actions of AFP, did not show a masculinized pattern of AVP expression (Bakker et al., 2006).

Sex differences in steroid responsiveness of AVP-expressing cells have also been reported in adulthood (De Vries et al., 1994b). Treatment of 3 month old rats with estradiol (E2) significantly stimulated AVP mRNA expression in both sexes, but significantly more so in males (De Vries et al., 1994b). DHT treatment by itself did not clearly stimulate AVP mRNA expression, but when given in combination with E2, it significantly increased the number of cells over that of animals treated with E2 alone. However, this increase was seen in males only. These studies in rats thus suggest that brain aromatization may be important in controlling the role of steroid hormones in the hypothalamic/limbic vasopressin system. Indeed, we recently showed that male mice which carry a mutation in the Cyp19 gene encoding the aromatase enzyme (aromatase knockout or ArKO; Honda et al., 1998) and as a result cannot convert androgens into estrogens, had fewer AVP-immunoreactive (ir) structures in the LS, BNST and Me than wild-type males (Plumari et al., 2002). Since only gonadally intact mice were studied in that particular study, it could not be determined whether the reduction in AVP expression in ArKO mice was due to the absence of any organizational or activational effects of estradiol on the AVP system.

Therefore in the present study, we determined the expression of AVP in the LS, BNST and Me of male ArKO mice which were treated with EB and dihydrotestosterone propionate (DHTP) in adulthood. We recently observed that this steroid hormone treatment was successful in stimulating male copulatory behaviors in male ArKO mice (Bakker et al., 2004) emphasizing the importance of estradiol signaling to the activational, as opposed to the organizational, effects of steroids on brain mechanisms controlling male-typical behaviors.

In addition, we determined whether this reduction in AVP expression in male ArKO mice is associated with deficits in social recognition. Site-specific injections of AVP into the LS of male rats resulted in a facilitation of social recognition by prolonging the time over which the memory was held (Engelmann and Landgraf, 1994). Previous results obtained in male ArKO mice have indicated that they do not show a conditioned place preference when estrous female stimuli are used (Pierman et al., 2006b). The absence of a conditioned place preference is most likely not due to any deficits in olfactory recognition per se, since male ArKO mice are perfectly capable to discriminate between different types of urine odors (Pierman et al., 2006a; Wesson et al., 2006). However, it may indicate certain deficits in social recognition. Therefore, we determined social discrimination in gonadally intact male ArKO mice using two different tests. First we used the classic social recognition test as reviewed in Bielsky and Young (2004) and second we used the production of ultrasonic vocalizations as a measure of olfactory recognition. Male mice emit $70 \mathrm{kHz}$ ultrasonic vocalizations under several conditions, mainly during courtship and copulation (Sales, 1972; Whitney et al., 1973). Although females have the capacity to emit these calls, and do so under certain circumstances (Maggio and Whitney, 1985), females rarely call in male-female pairings (Whitney et al., 1973). As a result, male mice produce more vocalizations than females and the stimulation of male calling is highly specific to females or their odors (Dizinno et al., 1978; Nyby et al., 1977). Finally, we determined whether adult treatment with EB and DHTP would correct any deficits in their social recognition.

\section{Materials and methods}

\section{Animals}

Aromatase knockout mice were generated by targeted disruption of exons 1 and 2 of the Cyp 19 gene (Honda et al., 1998). Heterozygous (HET) males and females of the $C 57 B l / 6 j$ strain were bred to generated wild-type (WT), HET and homozygous-null (ArKO) offspring. Mice were genotyped by PCR analysis of tail DNA (for more detailed description see Bakker et al., 2002). All breeding and genotyping were performed at the Center for Cellular and Molecular Neurobiology, University of Liège, Belgium. Food and water were always available ad libitum and the temperature was maintained at $22^{\circ} \mathrm{C}$. Subjects were housed alone under a reversed light-dark cycle (12:12 LD).

In the behavioral part of this study, a group of WT $(n=10)$ and $\operatorname{ArKO}(n=9)$ males was first tested for social recognition when gonadally intact. Then they were castrated and tested again for social recognition after 2 weeks of daily subcutaneous injection (s.c.) with dihydrotestosterone-proprionate (DHTP; $500 \mu \mathrm{g}$ in $0.05 \mathrm{ml} /$ mouse) and estradiol benzoate (EB; $5 \mu \mathrm{g}$ in $0.05 \mathrm{ml} /$ mouse). Ultrasonic vocalizations were recorded in another group of WT $(n=22)$ and ArKO $(n=21)$ males. Half of them were tested gonadally intact whereas the other half was castrated and treated daily for 2 weeks with DHTP ( $500 \mu \mathrm{g}$ in $0.05 \mathrm{ml} /$ mouse $)$ and EB ( $5 \mu \mathrm{g}$ in $0.05 \mathrm{ml} /$ mouse) by subcutaneous injection (s.c.) in the neck. Castration occurred under general anesthesia through an intraperitoneal injection (i.p.) of a mixture of ketamine $(80 \mathrm{mg} / \mathrm{kg}$ per mouse) and medetomidine (Domitor, Pfizer, $1 \mathrm{mg} / \mathrm{kg}$ per mouse). Mice received atipamezole (Antisedan, Pfizer, $4 \mathrm{mg} /$ $\mathrm{kg}$ per mouse, s.c.) at the end of the surgery in order to antagonize medetomidineinduced effects, thereby accelerating their recovery. All subjects were tested randomly between 10:00 $\mathrm{h}$ and 18:00 $\mathrm{h}$ during the dark phase of the LD cycle.

Finally, immunohistochemistry was conducted on brains from a new group of WT $(n=6)$ and ArKO $(n=6)$ males, all gonadally intact. Half of them (3 WT and 3 ArKO) were treated daily for 2 weeks with DHTP $(500 \mu \mathrm{g}$ in $0.05 \mathrm{ml} /$ mouse) and $\mathrm{EB}(5 \mu \mathrm{g}$ in $0.05 \mathrm{ml} / \mathrm{mouse})$ by s.c. injection in the neck whereas the other 3 WT and 3 ArKO received no additional hormonal treatment.

All experiments were conducted in accordance with the guidelines set forth by the National Institutes of Health Guiding Principles for the Care and Use of Research Animals and were approved by the Ethical Committee for Animal Use of the University of Liège. 


\section{Social recognition test}

A social habituation-dishabituation paradigm, reviewed in Bielsky and Young (2004), was used to evaluate social recognition abilities in WT and ArKO males. This test used the animals' natural tendency to investigate novel stimuli. Males of both genotypes were housed individually for at least 1 week before testing. On the test day, males were repeatedly exposed to an unfamiliar ovariectomized female placed in their cage for $1 \mathrm{~min}$ during four successive trials and the time spent by the male in active and direct olfactory investigation was recorded (including head and anogenital sniffing as well as close following and pursuit). During the trial-intervals $(10 \mathrm{~min})$ the female was removed and the male stayed alone in their cage. Normally, repeated presentations of the same stimulus will lead to a marked decrease (habituation) in the amount of time spent in olfactory investigation, as it becomes familiar. On the fifth trial, males were divided in two groups depending on whether they were exposed to the same ( $5 \mathrm{WT}$ and $5 \mathrm{ArKO}$ ) or to a new female ( $5 \mathrm{WT}$ and $4 \mathrm{ArKO}$ ). In the last case, the time spent investigating the stimulus female should increase if the male recognizes the female as unfamiliar.

This social recognition test was conducted first in gonadally intact WT and ArKO males. Then they were then castrated and treated daily with EB and DHTP for 2 weeks before being tested again for social recognition. By doing so, we should be able to demonstrate whether adult estradiol treatment would correct any possible deficits in the social recognition abilities of ArKO males.

\section{Recording of ultrasonic vocalizations}

Ultrasonic vocalizations (UV) were recorded in WT and ArKO males which were either gonadally intact or castrated and treated daily with EB and DHTP. A sequence of 4 stimuli (in random order) was used to induce ultrasonic vocalizations in each animal: intact male urine, estrous female urine, the presence of an anesthetized male or an anesthetized gonadectomized female implanted with a silastic capsule containing crystalline estradiol and injected with progesterone 2 to $4 \mathrm{~h}$ before testing. Urine stimuli were presented by pipetting $100 \mu \mathrm{l}$ onto a piece of filter paper that was introduced into the cage of each subject, while stimulus animals were anesthetized using the same mixture as used for castration (i.p. injection of a mixture of ketamine and medetomidine) and were placed in the cage of the subject. $70 \mathrm{~Hz}$ ultrasonic vocalizations emitted in response to the stimulus were recorded during $5 \mathrm{~min}$ (per stimulus) using UltraVox detector (Noldus). The total duration of ultrasonic vocalizations allowed us to evaluate the response of the subject toward the stimulus and was used as dependent value in the statistical analysis.

\section{Colchicine injections for AVP visualization}

In order to better visualize AVP-immunoreactive (ir) cell bodies, a third group of WT and ArKO males ( $n=6$ for each genotype), half of them treated with EB and DHTP for at least 2 weeks, whereas the other half remained untreated, were anesthetized with a mixture of ketamine (173 $\mu \mathrm{g} / \mathrm{g}$ mouse) and xylazine $(6 \mu \mathrm{g} / \mathrm{g}$ mouse) and placed in a stereotaxic frame (Kopf, Tujunga, CA, USA). The mice were stereotaxically injected with $5 \mu$ of a colchicine solution $(6 \mathrm{mg} / \mathrm{ml})$ at $0.2 \mu \mathrm{l} / \mathrm{min}$ in the lateral ventricle. The accuracy of the position of the injection cannula was controlled by observing the increase in pressure in the cannula when it entered the ventricle. Later inspection of the brain sections did not reveal any trace of damaged tissue around the lateral ventricle, except for where the needle entered the ventricle. Two days following the colchicine injection, all mice were deeply anesthetized with ketamine and xylazine and intracadiacally perfused with $4 \%$ paraformaldehyde containing $0.1 \%$ glutaraldehyde in $100 \mathrm{ml}$ phosphate buffer (PB; pH: 7.2). Brains were removed and postfixed in paraformaldeyhyde/glutaraldehyde for $2 \mathrm{~h}$. Then brains were cryoprotected in $30 \%$ sucrose/PBS and when sunken, frozen on dry ice and shipped to Torino where they were stored at $-70{ }^{\circ} \mathrm{C}$ until used for immunohistochemistry.

\section{Immunohistochemistry}

Brains were cut in the coronal plane with a cryostat at a thickness of $25 \mu \mathrm{m}$. Every fourth section was stained for AVP by immunocytochemistry, the other sections were stored in an antifreeze solution at $-20{ }^{\circ} \mathrm{C}$. Free-floating sections were immunostained by avidin-biotin system using a commercial AVP antibody (ICN Pharmaceuticals, CA, USA) at the dilution of 1:20,000. The specificity of this antibody to vasopressin (and thus the absence of any cross-reactivity with oxytocin) has been validated previously (Ferris et al., 1995). However, in the present study, in order to control for staining specificity, several control incubations were performed in which the primary antibody or the secondary one was replaced by normal goat serum. No signal was observed in these conditions. A second series of brain sections was stained using toluidine blue for anatomical orientation. All structures were identified using the atlas of the mouse brain (Franklin and Paxinos, 1997).

\section{Staining analysis}

All sections were first observed with a light microscope (Olympus BH-2, Olympus Italia, Milano, Italy). Selected fields were then acquired by a CCDcamera (Hamamatsu C 4200, Hamamatsu Italia, Milano, Italy) connected to a Power PC Macintosh 4400 equipped with a digitizing board. The staining density of AVP-ir containing structures was measured in selected nuclei with the NIH Image program (version 1.62, a public domain program written by W. Rasband at the US National Institute of Health, Bethesda, MD, USA) by calculating in binary transformations of the images the fractional area (percentages of pixels) covered by the structures in predetermined fields as described in previous publications (Plumari et al., 2002; Viglietti-Panzica et al., 1994, 2001). Two rostro-caudal levels (anterior and posterior) were studied in the LS (levels corresponding to stereotaxic coordinates 0.62 and 0.14 relative to Bregma in the mouse brain atlas) and $\mathrm{Me}(-1.22$ and -1.70 relative to Bregma) to control for a possible heterogeneity of AVP distribution in these large structures. The fractional area covered by AVP-ir structures was determined in the same way in the BNST $(-0.22$ relative to Bregma) and the supraoptic nucleus ( $\mathrm{SON} ;-0.82$ relative to Bregma)

\section{Statistical analysis}

In the social recognition tests, time spent investigating the female during the five successive tests was used as dependent factor in a two-way ANOVA adapted for repeated measures. Genotype (WT vs ArKO) and group (same or new female presented during the fifth trial) were used as independent factors. This statistical analysis was done on gonadally intact and castrated and EB +DHTP treated males separately. When appropriate, post-hoc comparisons were carried out by Fisher Least Signification Difference (LSD) post-hoc comparisons.

$T$-tests for repeated measures were used to compare the UV total duration in response to male versus female stimulus for each group of subjects (depending of their genotype and hormonal status). By doing so, we were able to detect whether UV were (or not) oriented toward a sex-specific stimulus (male versus female) in each group.

Finally, AVP-ir staining intensities (expressed by the fractional area covered by AVP-ir fibers) were analyzed by two-way ANOVAs with genotype and hormonal status as independent factors. When appropriate, post-hoc comparisons were carried out for each brain area by Fisher Least Signification Difference (LSD) post-hoc comparisons. $P<0.05$ was considered statistically significant.

\section{Results}

\section{Social recognition test}

Gonadally intact WT males spent less time investigating the stimulus female following successive presentations as she becomes familiar (habituation). However, their investigation time increased again when a new female was presented on the last trial (dishabituation; Fig. 1A). By contrast, gonadally intact ArKO males did not show a clear habituation response when presented with the same female repeatedly. Overall, they continued to investigate the stimulus female and when presented with the same or new female on the fifth trail did not change their investigation times significantly (no dishabituation; Fig. 1A). 
A

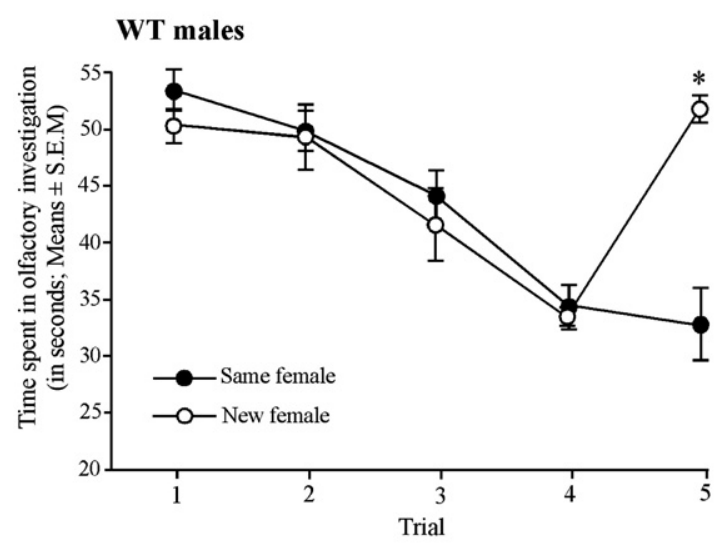

Gonadally intact males

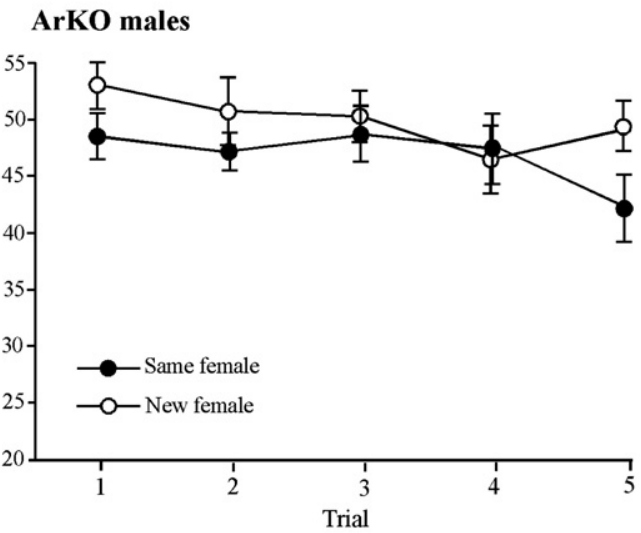

B

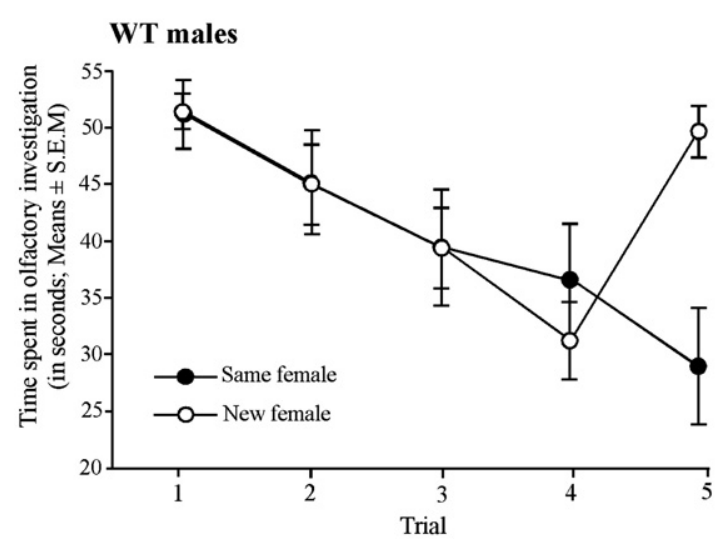

Castrated males treated with EB and DHTP

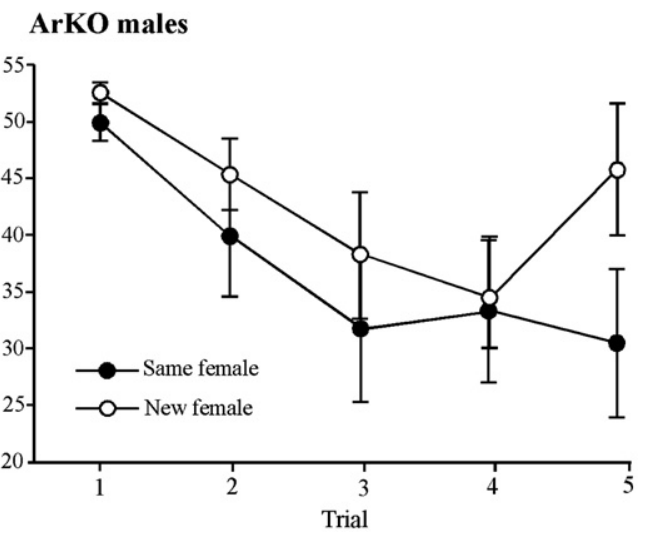

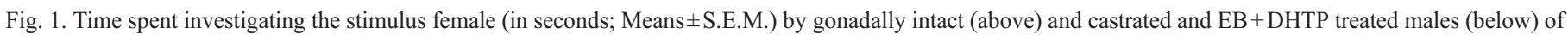

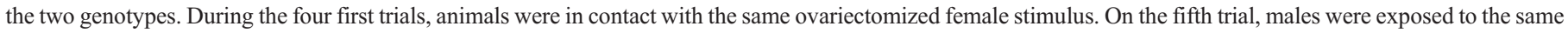
(black circle) or to a new female (open circle). $P<0.05 *$ different from the time spent in olfactory investigation by the same group on the fourth trial.

Two-way ANOVA on the time spent in olfactory investigation during the five trials by gonadally intact WT and ArKO males revealed a significant effect of genotype $(F(1,15)=5.478$, $P=0.03)$, group $(F(1,15)=4.696, P=0.047)$, trial $(F(4,60)=$ $19.068, P<0.0001)$ and a significant trial by group interaction $(F(4,60)=8.765, P<0.0001)$. Trial by genotype $(F(4,60)=8.94$, $P<0.0001)$ and trial by group by genotype $(F(4,60)=3.6, P=$ 0.011 ) interactions were also significant.

Post-hoc analysis showed that the time spent investigating the stimulus female was reduced between the first and the fourth presentation of the same female in gonadally intact WT males (trial 1 vs trial 4: "same female" group: $P<0.0001$ and "new female" group: $P<0.0001$ ) whereas this reduction was less obvious in gonadally intact ArKO males (trial 1 vs trial 4: "same female" group: $P=0.74$ and "new female" group: $P=0.02$ ). Furthermore, at the fourth presentation of the female stimulus, ArKO males spent significantly more time investigating the stimulus female than WT males (investigation time on the fourth trial: WT vs ArKO of the "same female" group: $P=0.0004$; WT vs ArKO of the "new female" group: $P=0.0006$ ) suggesting that ArKO males did not habituate like WT males.

Finally, the investigation time on the last trial was markedly increased and restored to the level observed initially when a new female (but not the same female) was introduced to gonadally intact WT males (trial 4 vs trial 5: "new female" group: $P<$ 0.0001; "same female" group: $P=0.6$ ). Interestingly, the time spent investigating in the fifth trial did not increase in gonadally intact ArKO males when a new female was introduced (trial 4 vs trial 5: "new female" group: $P=0.3$; "same female" group: $P=0.09$ ).

By contrast, following castration and daily treatment with EB and DHTP, ArKO males, like WT males habituated to the presentation of the same female (Fig. 1B). Thus, investigation times decreased in both genotypes following successive presentations of a same female as she became familiar and were restored to the levels observed initially when a new female was presented on the fifth trial (dishabituation).

Two-way ANOVA on the time spent investigating the female stimulus during the five trials by castrated and EB +DHTPtreated WT and ArKO males only revealed a significant effect of trial $(F(4,60)=27.96, P<0.0001)$ and a group by trial interaction $(F(4,60)=9.03, P<0.0001)$. Interestingly, the effect of genotype and any interactions involving the genotype were no longer significant suggesting that social recognition abilities of EB + DHTP-treated ArKO males were similar to those of similarly treated WT males. Post-hoc analysis showed that the time 
spent in investigating by castrated and EB + DHTP treated males (both genotypes taken together) was reduced between the first and the fourth presentation of the same female (trial 1 vs trial 4 : "same female" group: $P<0.0001$ and "new female" group: $P<$ 0.0001). Furthermore, the investigation time on the last trial was markedly increased and nearly restored to the initial level when a new female (but not the same female) was presented to these males ("new female" group: trial 4 vs trial 5: $P<0.0001$ and trial 1 vs trial 5: $P=0.09$; "same female" group: trial 4 vs trial 5: $P=0.042$ and trial 1 vs trial 5: $P<0.0001)$.

\section{Ultrasonic vocalizations}

Gonadally intact WT males produced ultrasonic vocalizations directed towards stimuli coming from an estrous female. This preference was not so evident when exposed to urine (Fig. 2A; intact WT males: UV in response to male vs female urine: $P=0.09$ ), but was more obvious when an anesthetized conspecific was introduced in their home cage (Fig. 2B; intact WT males: UV in response to anesthetized male vs female: $P=0.002$ ). By contrast, gonadally intact ArKO males also produced ultrasonic vocalizations but, remarkably, they vocalized as much for the male as for the female stimuli (Figs. 2A and B;
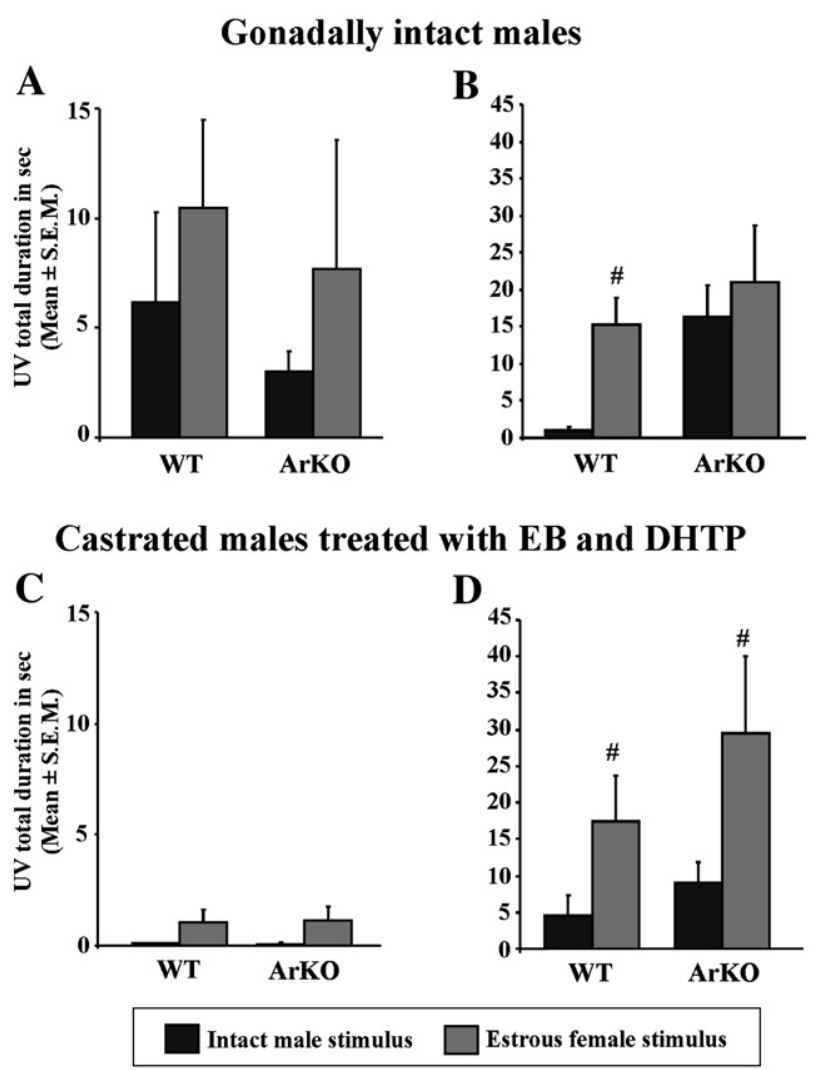

Fig. 2. Total duration of ultrasonic vocalizations (in seconds; Means \pm S.E.M.) emitted by gonadally intact (above; $\mathrm{WT}=11$ and $\mathrm{ArKO}=10$ ) and castrated and $\mathrm{EB}+\mathrm{DHTP}$ treated males of both genotypes (below; 11 males for each genotype) when exposed to stimuli derived from intact males (black bar) or estrous females (grey bar). Vocalizations were recorded when exposed to urinary odors $(\mathrm{A}$ and $\mathrm{C}$ ) or when presented with an anesthetized conspecific (B and D). $P<0.05$ \#different from the same group exposed to the male stimulus.
Table 1

Summary of the two-ways ANOVA on the fractional area covered by AVP-ir fibers in several brain regions of gonadally intact and EB+DHTP treated WT and ArKO males

\begin{tabular}{|c|c|c|c|c|c|c|}
\hline & \multicolumn{6}{|c|}{ Statistical effects } \\
\hline & \multicolumn{2}{|c|}{$\begin{array}{l}\text { Genotype } \\
\text { df (1.8) }\end{array}$} & \multicolumn{2}{|c|}{$\begin{array}{l}\text { Hormonal status } \\
\text { df (1.8) }\end{array}$} & \multicolumn{2}{|c|}{$\begin{array}{l}\text { interaction } \\
\text { df (1.8) }\end{array}$} \\
\hline & $F$ & $P$ & $F$ & $P$ & $F$ & $P$ \\
\hline LS1 & 3,7 & 0.09 & 18,5 & 0.002 & 0,15 & 0.71 \\
\hline LS2 & 4,03 & 0.08 & 14,74 & 0.005 & 8,84 & 0.02 \\
\hline $\mathrm{MeA}$ & 2,38 & 0.16 & 5,19 & 0.05 & 1,56 & 0.24 \\
\hline $\mathrm{MeP}$ & 30,66 & $<\mathbf{0 . 0 0 1}$ & 12,82 & 0.007 & 1,06 & 0.33 \\
\hline BST & 0,29 & 0.6 & 0,38 & 0.55 & 6,6 & 0.03 \\
\hline SON & 5,13 & 0.05 & 0,24 & 0.64 & 0,66 & 0.44 \\
\hline
\end{tabular}

$p<0.05$ in bold.

intact ArKO males: UV in response to male vs female urine: $P=0.34$; to anesthetized male vs female: $P=0.44$ ). So, gonadally intact ArKO males did not seem to produce sexuallyoriented ultrasonic vocalizations (Fig. 2A and B).

When castrated and treated daily with EB and DHTP, both WT and ArKO males emitted few vocalizations in response to male and female urine (Fig. $2 \mathrm{C}$; UV to male vs female urine: WT: $P=0.06$ and ArKO: $P=0.11$ ) but interestingly, ArKO males now produced predominantly ultrasonic vocalizations in response to an anesthetized estrous female compared to the male (Fig. 2D; UV in response to anesthetized male vs female: WT: $P=0.03$ and ArKO: $P=0.05$ ). Thus their vocalizations towards conspecifics became sexually-oriented when ArKO males were supplemented with EB and DHTP in adulthood (Figs. 2C and D).

\section{AVP immunochemistry}

In gonadally intact WT males, the distribution of AVPimmunoreactivity was in line with what has previously been described in the mouse and the rat (Plumari et al., 2002; De Vries et al., 1994b). Thus, a clear vasopressinergic innervation of the LS was observed in gonadally intact WT but not so in ArKO males. Likewise, the BNST and the Me were more densely innervated by AVP-ir structures in WT than in ArKO males. These results confirm our previous observations (Plumari et al., 2002) of a diminished AVP-immunoreactivity in sexually dimorphic structures in male ArKO mice. Remarkably adult treatment with EB and DHTP induced a significant increase in AVP expression in the caudal LS and, although less obvious, in the Me and the BNST of ArKO males. Statistical results of the two-way ANOVAs on the fractional area covered by AVP-ir structures in the different brain regions investigated are summarized in Table 1.

Thus, two-way ANOVA on the AVP-ir expressed in the whole LS (AVP-ir in the rostral and caudal LS as repeated measures) revealed a significant effect of genotype $(F(1,8)=$ 13.29, $P=0.006)$, of hormonal treatment $(F(1,8)=54.29, P<$ $0.0001)$ and a significant genotype by hormonal treatment interaction $(F(1,8)=10.7, P=0.01)$. Post-hoc analysis showed that AVP expression was reduced in the LS of intact ArKO males 
compared to WT $(P=0.001)$ and that adult treatment with EB and DHTP increased AVP expression in ArKO males to the levels observed in WT males (intact vs treated ArKO males: $P<0.0001$; WT vs ArKO treated males: $P=0.8)$. There was also an effect of the region (rostral vs caudal; $F(1,8)=20,52$, $P=0.002$ ) thereby showing that AVP expression was much higher in the caudal (LS2) than in the rostral (LS1) lateral septum. In fact, separated ANOVAs for each region of the LS showed that the genotype by hormonal treatment interaction was only significant for the caudal $\operatorname{LS}(F(1,8)=8.84, P=0.02)$. Indeed, AVP expression in the caudal LS (LS2) was reduced in ArKO compared to WT intact males $(P=0.008)$ and was restored to the level of WT males by hormonal treatment (WT vs ArKO treated males: $P=0.54$; Figs. 3 and 4). In the rostral LS (LS1), AVP expression was weak and did not differ significantly between WT and ArKO intact males, but was increased following EB and DHTP treatment in both genotypes (effect of the hormonal treatment: $F(1,8)=18.5, P=0.002)$.

AVP expression in the Me (Fig. 4) was reduced in ArKO males, especially in the MeP (effect of the genotype: $F(1,8)=$ 30.66, $P<0.001)$. Furthermore, EB and DHTP treatment increased AVP expression in the MeA and the MeP (effect of the hormonal treatment was significant for both areas; see Table 1) but statistical results did not allow us to confirm that this increase was significantly different between WT and ArKO males since there was no significant genotype by hormonal treatment interaction $(\mathrm{MeA}: F(1,8)=1.56, P=0.24$; MeP: $F(1,8)=1.06, P=0.33)$.
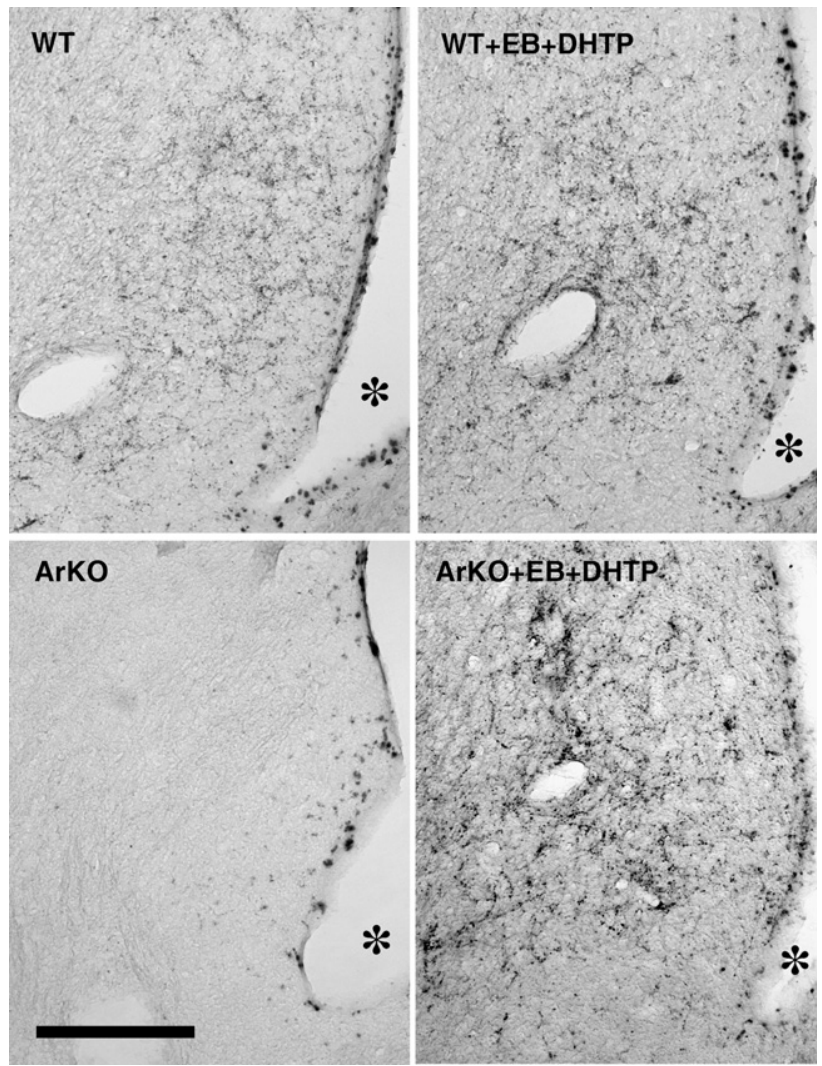

Fig. 3. Representative photomicrographs showing AVP-immunoreactive staining in the caudal lateral septum (LS2) of gonadally intact and EB + DHTPtreated WT and ArKO males. The scale bar represents $200 \mu \mathrm{m}$.

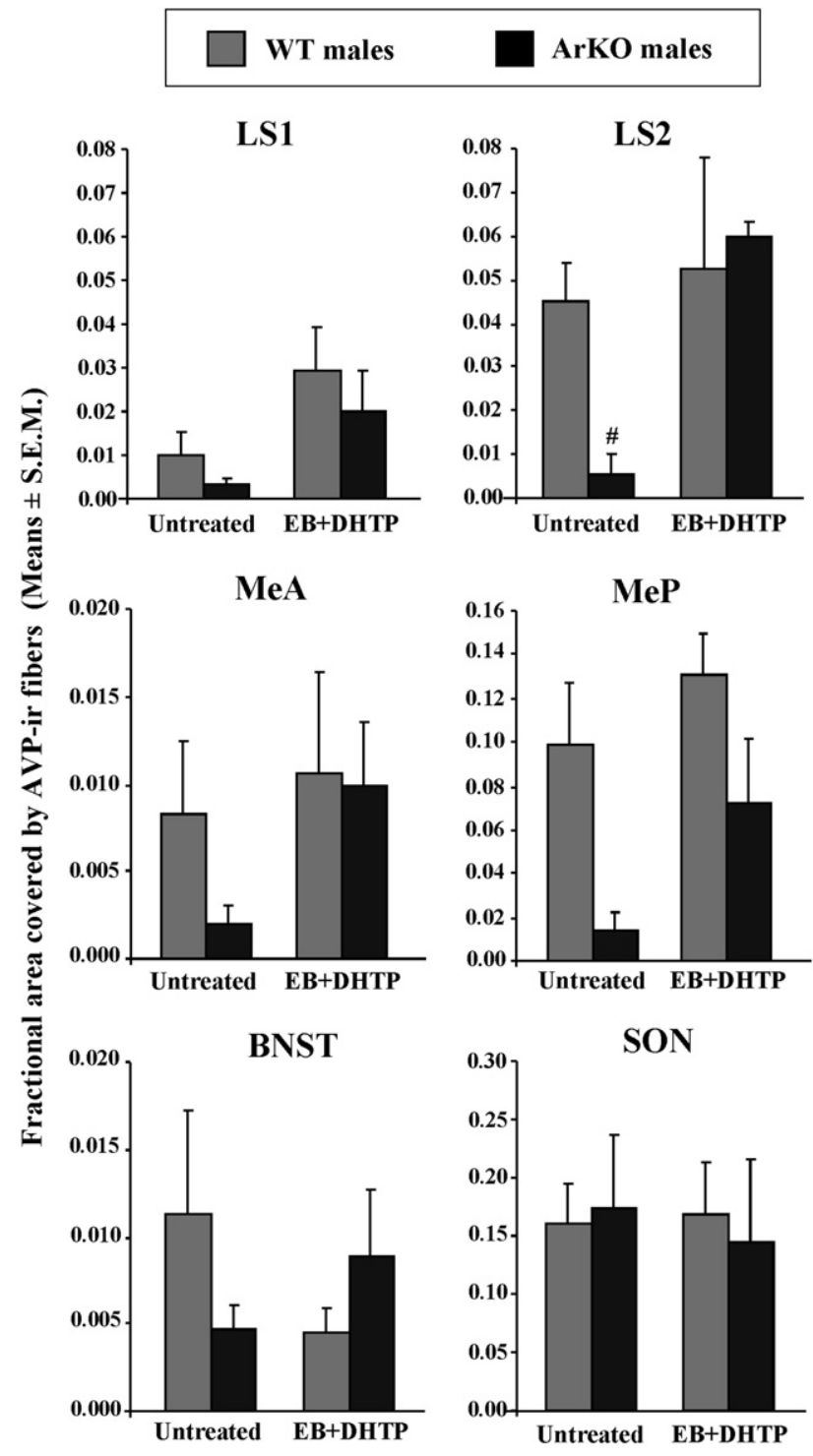

Fig. 4. Fractional area covered by AVP-ir fibers (means \pm S.E.M.) in the rostral (LS1) and caudal (LS2) lateral septum, in the anterior (MeA) and posterior (MeP) medial amygdala, in the BNST and SON of WT and ArKO males. Mice were gonadally intact and treated or not with EB and DHTP in adulthood (3 WT and 3 ArKO males in each group). $P<0.05$ \#different of WT males in the same condition.

Statistical analysis of the AVP expression observed in the BNST (Fig. 4) showed a significant genotype by hormonal treatment interaction $(F(1,8)=6 \cdot 6, P=0.03)$. AVP expression was reduced in the BNST of gonadally intact ArKO males compared to WT gonadally intact males, although not clearly significant $(P=0.054)$ and was partially restored in ArKO males following treatment with exogenous hormones (treated WT vs ArKO males: $P=0.20)$.

Finally, statistical analysis on the AVP expression observed in the SON, one possible source of AVP production for peripheral release, did not show any significant effect or interaction. No difference in AVP expression were observed between gonadally intact WT and ArKO males whatever they were treated with EB + DHTP in adulthood or not (Fig. 4). 


\section{Discussion}

The present study shows that estradiol has strong activational effects on socio-sexual recognition in mice. Gonadally intact ArKO males produced ultrasonic vocalizations when presented with an anesthetized conspecific but unlike WT males, their vocalizations were not directed preferentially toward the female stimulus. These results are consistent with previous studies showing that ArKO males did not investigate female odors preferentially in a Y maze (Bakker et al., 2002). Moreover, results of the social recognition test suggested that intact ArKO males were not able to recognize a 'familiar' female since their investigation time did not decrease with successive presentations of a female stimulus. Thus it seems that gonadally intact ArKO males are not able to recognize a potential mate and therefore may not adjust their behavior toward females. This may explain the strong deficits affecting copulatory behavior of ArKO males (Bakker et al., 2004).

Accordingly, a decrease in AVP expression, a neuropeptide that has been implicated in social recognition in rodents (Bielsky and Young, 2004) was observed in intact ArKO males (as previously shown by Plumari et al., 2002). This decrease in AVP expression was most obvious in the lateral septum, a region which is critical for social recognition and memory (Engelmann et al., 1996; Wimersma Greidanus, 1982; Wimersma Greidanus et al., 1983). For instance, the impairment of social recognition that is observed in male Brattleboro rats which lack AVP could be rescued by administration of AVP into the lateral septum by microdialysis (Engelmann and Landgraf, 1994). In addition, male mice lacking functional vasopressin 1a receptors (V1aRKO) exhibit markedly reduced anxiety like behavior and a profound impairment in social recognition (Bielsky et al., 2004) thereby providing strong evidence for a physiological role of AVP in this region for social recognition. We thus demonstrated here that the absence of estrogens disturbs social recognition in male mice and that these deficits were visible at the behavioral as well as the cellular level.

Our finding of clear activational effects of estrogens on social recognition in male mice may seem at odds with the findings of Bluthe et al. (1990). They showed that social recognition was disrupted in castrated male rats 1 week after the surgery, but that it was restored 2 weeks later. The temporary disruption in social memory was interpreted as "a gradual shift from a vasopressinergic mediated neurotransmission to a non-vasopressinergic one in the neural circuit(s) involved in social recognition, following the drop in plasma testosterone levels due to castration" (Bluthe et al., 1990). Furthermore, the finding that the V1 antagonist blocked social recognition in sexually intact but not in castrated male and intact female rats is consistent with the interpretation that androgen-dependent vasopressin circuitry plays a significant role in mediating social recognition in sexually intact adult male rats, but not in castrated male and intact female rats (Bluthe and Dantzer, 1990). However, it should be noted that the animals in the experiments of Bluthe et al. (1990) were castrated when the behavior was already developed (they have tested the behavior before the animals were castrated), so their males developed their vasopressin system under normal con- ditions (i.e. gonad-intact and with androgens and estrogens). By contrast, ArKO males have never been exposed to estrogens throughout life thus it is unpredictable whether other neural circuits that are associated with the AVP system in regulating social recognition are intact (if estrogen-dependent, they have not been developed normally, if estrogen-independent, they are essentially intact). Thus the experiments of Bluthe et al. (1990) are dealing primarily with the activational effects of androgens on a system, i.e. the AVP system of the rat, which is essentially intact, whereas we are dealing with an intact system (the AVP one) which is included in a variety of altered neural circuits. Furthermore, estradiol can also come from extragonadal sources in castrated male rats. The adrenal androgen DHEA can enter the brain and be converted to androstendione-testostosteroneestradiol. Thus it is possible that the social recognition abilities were restored in castrated male rats by estrogens coming from adrenal sources. Obviously this is not the case in ArKO mice since they cannot convert androgens to estrogens.

Adult treatment with EB and DHTP induced a WT male-like AVP expression in male ArKO mice which suggests that the previously observed (Plumari et al., 2002) decrease in AVP expression in gonadally male ArKO mice was largely due to the absence of activational rather than organizational effects of estradiol in these mice. This was less clear with regard to the BNST and the Me (where hormonal treatment increased AVP expression in both genotypes) but was most obvious in the caudal lateral septum receiving projections from these brain regions, where adult treatment with EB and DHTP fully restored AVP expression in ArKO mice at similar levels than those normally observed in WT males. Thus, the AVP system of male ArKO mice, especially the lateral septum, can still be activated by exogenous hormones in adulthood which clearly indicates that the decrease observed in gonadally intact subjects is not the result of irreversible organizational effects such as an induction of neuronal death due to the lack of estrogens during ontogeny, but is rather due to a lack of activation by steroids in adulthood.

This idea of estrogens not having any organizational actions on the AVP system in mice is in line with previous studies on alpha-fetoprotein knockout mice (AFP-KO). Indeed, AFP-KO females, exposed to a large amount of maternal estrogens during development since they are not protected by alpha-fetoprotein, an important fetal plasma protein that binds estrogens with high affinity, did not show a male-typical AVP system. These results suggest that estrogens have probably no organizational, masculinizing effect on this sexually dimorphic neuropeptide system in mice (Bakker et al., 2006) whereas both estrogenic and androgenic metabolites seem to have organizational effects on this system in rats (Han and De Vries, 2003). Alternatively, the development of the sexually dimorphic AVP system may require the presence of androgens (Allieri et al., 2005) or the direct contribution of sex chromosomes (De Vries et al., 2002). Indeed, in male rats with the testicular feminization mutation (spontaneous mutants lacking a functional androgen receptor) the AVP system in the BNST, MeA and MeP is significantly reduced (Allieri et al., 2005) whereas XY males and XY females (that is, genetic males with ovaries due to a deletion of the Sry gene, which encodes the testes-determining factor) are more 
masculine than XX mice with regard to the density of AVPexpressing fibers in the lateral septum (De Vries et al., 2002).

Interestingly, the finding that adult treatment with $\mathrm{EB}$ and DHTP was also able to restore sexually-oriented production of ultrasonic vocalizations as well as social recognition in ArKO males is perfectly in line with our finding of a restored AVP system in these males. Thus EB- and DHTP-treated ArKO males expressed sexually directed ultrasonic vocalizations while this was not the case when gonadally intact. Furthermore, EB- and DHTP-treated ArKO males showed a clear habituation response when exposed to the same female and a dishabituation response when exposed to a new female, as was observed in WT males. Thus estrogens have activational effects on both the AVP system and social recognition suggesting that they are closely associated. However, further experiments will be necessary to confirm that the deficits in social recognition observed in gonadally intact ArKO males clearly reflect a dysfunction of the AVP circuitry by investigating, for example, the effects of central administration of AVP on the social memory abilities of these mice. Finally, the possibility cannot be excluded that reinstatement of social recognition and AVP expression in the lateral septum of ArKO males following EB+DHTP treatment could also be due to activational effects of DHTP rather than $\mathrm{EB}$; this is probably not the case since gonadally intact ArKO males presumably have similar or even higher levels of DHT than WT males since Fisher et al. (1998) showed that testosterone levels were increased in ArKO males since they lack the estrogen feedback action on the hypothalamic-pituitary axis.

In summary, the present study shows that the steroid-sensitive sexually dimorphic AVP system of the mouse forebrain and social recognition abilities are affected by activational effects of predominantly the estrogenic metabolite of testosterone. Moreover, our results are in line with the idea that organization of these neuropeptide system during development may depend on androgen or sex chromosomes rather than estrogens.

\section{Acknowledgments}

This work was supported by the National Institutes of Health Grant (HD 044897) and by the Belgian "Fonds National de la Recherche Scientifique (FNRS)" to Sylvie Pierman (FNRS Research Fellow) and Dr. Julie Bakker (FNRS Research Associate; FNRS 1.5.104.06) and by COFIN 2006 (2006072719_003 GCP), the University of Torino (GCP and CVP) and Fondazione CRT (GCP) to Pr. Giancarlo Panzica.

\section{References}

Allieri, F., Spigolon, G., Viglietti-Panzica, C., Garcia-Falgueras, A., Guillamon, A., Collado, P., Panzica, G.C., 2005. The Tfm rat, a model to study the influence of testosterone on the development of limbic vasopressinergci system. Trabajos del Instituto Cajal LXXX, 198-199.

Bakker, J., Honda, S., Harada, N., Balthazart, J., 2002. Sexual partner preference requires a functional aromatase (Cyp19) gene in male mice. Horm. Behav. $42,158-171$.

Bakker, J., Honda, S., Harada, N., Balthazart, J., 2004. Restoration of male sexual behavior by adult exogenous estrogens in male aromatase knockout mice. Horm. Behav. 46, 1-10.
Bakker, J., De Mees, C., Douhard, Q., Balthazart, J., Gabant, P., Szpirer, J., Szpirer, C., 2006. Alpha-fetoprotein protects the developing female mouse brain from masculinization and defeminization by estrogens. Nat. Neurosci. 9, 220-226.

Bielsky, I.F., Young, L.J., 2004. Oxytocin, vasopressin, and social recognition in mammals. Peptides 25, 1565-1574.

Bielsky, I.F., Hu, S.B., Szegda, K.L., Westphal, H., Young, L.J., 2004. Profound impairment in social recognition and reduction in anxiety-like behavior in vasopressin V1a receptor knockout mice. Neuropsychopharmacology 29, 483-493.

[ROMAN]Bluthe, R.M., Dantzer, R., 1990. Social recognition does not involve vasopressinergic neurotransmission in female rats. Brain Res. 535, 301-304.

Bluthe, R.M., Schoenen, J., Dantzer, R., 1990. Androgen-dependent vasopressinergic neurons are involved in social recognition in rats. Brain Res. 519, $150-157$.

Brown, R.E., 1979. Mammalian social odors: a critical review. Adv. Study Behav. 10, 107-161.

De Vries, G.J., Buijs, R.M., 1983. The origin of the vasopressinergic and oxytocinergic innervation of the rat brain with special reference to the lateral septum. Brain Res. 273, 307-317.

De Vries, G.J., Al Shamma, H.A., 1990. Sex differences in hormonal responses of vasopressin pathways in the rat brain. J. Neurobiol. 21, 686-693.

De Vries, G.J., Boyle, P.A., 1998. Double duty for sex differences in the brain. Behav. Brain Res. 92, 205-213.

De Vries, G.J., Miller, M.A., 1998. Anatomy and function of extrahypothalamic vasopressin systems in the brain. Prog. Brain Res. 119, 3-20.

De Vries, G.J., Panzica, G.C., 2006. Sexual differentiation of central vasopressin and vasotocinsystems in vertebrates: different mechanisms, similar endpoints. Neuroscience 138, 947-955.

De Vries, G.J., Al Shamma, H.A., Zhou, L., 1994a. The sexually dimorphic vasopressin innervation of the brain as a model for steroid modulation of neuropeptide transmission. In: Harding, C.F. (Ed.), Hormonal restructuring of the adult brain. Basic and clinical perspectives. Annals, vol. 743. New York Academy of Sciences, New York, USA, pp. 95-120.

De Vries, G.J., Wang, Z., Bullock, N.A., Numan, S., 1994b. Sex differences in the effects of testosterone and its metabolites on vasopressin messenger RNA levels in the bed nucleus of the stria terminalis of rats. J. Neurosci. 14, 1789-1794.

De Vries, G.J., Rissman, E.F., Simerly, R.B., Yang, L.Y., Scordalakes, E.M., Auger, C.J., Swain, A., Lovell-Badge, R., Burgoyne, P.S., Arnold, A.P., 2002. A model system for study of sex chromosome effects on sexually dimorphic neural and behavioral traits. J. Neurosci. 22, 9005-9014.

Dizinno, G., Whitney, G., Nyby, J., 1978. Ultrasonic vocalizations by male mice (mus musculus) in response to a female-produced pheromone: effects of experience. Behav. Biol. 22, 104-113.

Engelmann, M., Landgraf, R., 1994. Microdialysis administration of vasopressin into the septum improves social recognition in Brattleboro rats. Physiol. Behav. 55, 145-149.

Engelmann, M., Wotjak, C.T., Neumann, I., Ludwig, M., Landgraf, R., 1996. Behavioral consequences of intracerebral vasopressin and oxytocin: focus on learning and memory. Neurosci. Biobehav. Rev. 20, 341-358.

Ferris, C.F., Delville, Y., Miller, M.A., Dorsa, D.M., De Vries, G.J., 1995. Distribution of small vasopressinergic neurons in golden hamsters. J. Comp. Neurol. 360, 589-598.

Fisher, C.R., Graves, K.H., Parlow, A.F., Simpson, E.R., 1998. Characterization of mice deficient in aromatase (ArKO) because of targeted disruption of the cyp19 gene. Proc. Natl. Acad. Sci. 95, 6965-6970.

Franklin, K.B.J., Paxinos, G., 1997. The mouse brain in stereotaxic coordinates. Academic Press, New York.

Han, T.M., De Vries, G.J., 2003. Organizational effects of testosterone, estradiol, and dihydrotestosterone on vasopressin mRNA expression in the bed nucleus of the stria terminalis. J. Neurobiol. 54, 502-510.

Honda, S., Harada, N., Ito, S., Takagi, Y., Maeda, S., 1998. Disruption of sexual behavior in male aromatase-deficient mice lacking exons 1 and 2 of the cyp 19 gene. Biochem. Biophys. Res. Commun. 252, 445-449.

Maggio, J.C., Whitney, G., 1985. Ultrasonic vocalizing by adult female mice (Mus musculus). J. Comp. Psychol. 99, 420-436. 
Nyby, J.G., Dizinno, G., Whitney, G., 1977. Sexual dimorphism in ultrasonic vocalizations of mice (Mus musculus): gonadal hormone regulation. J. Comp. Physiol. Psychol. 91, 1424-1431.

Pierman, S., Douhard, Q., Balthazart, J., Baum, M.J., Bakker, J., $2006 a$. Attraction thresholds and sex discrimination of urinary odorants in male and female aromatase knockout (ArKO) mice. Horm. Behav. 49, 96-104.

Pierman, S., Tirelli, E., Douhard, Q., Baum, M.J., Bakker, J., 2006b. Male aromatase knockout mice acquire a conditioned place preference for cocaine but not for contact with an estrous female. Behav. Brain Res. 174, $64-69$.

Plumari, L., Viglietti-Panzica, C., Allieri, F., Honda, S., Harada, N., Absil, P., Balthazar, J., Panzica, G.C., 2002. Changes in the arginine-vasopressin immunoreactive systems in male mice lacking a functional aromatase gene. J. Neuroendocrinol. 14, 971-978.

Sales, G.D., 1972. Ultrasound and mating behavior in rodents with some observations on other behavioral situations. J. Zool. 168, 149-164.
Viglietti-Panzica, C., Aste, N., Balthazart, J., Panzica, G.C., 1994. Vasotocinergic innervation of sexually dimorphic medial preoptic nucleus of the male Japanese quail: influence of testosterone. Brain Res. 657, 171-184.

Viglietti-Panzica, C., Balthazart, J., Fratesi, S., Plumari, L., Absil, P., Panzica, G.C., 2001. Estradiol mediates effects of testosterone on vasotocin-immunoreactivity in the adult quail brain. Horm. Behav. 40, 445-461.

Wesson, D.W., Keller, M., Douhard, Q., Baum, M.J., Bakker, J., 2006. Enhanced urinary odor discrimination in female aromatase knockout (ArKO) mice. Horm. Behav. 49, 580-586.

Whitney, G., Cable, J.R., Stockton, M.D., Tilson, E.F., 1973. Ultrasonic emissions: do they facilitate courtship of mice? J. Comp. Physiol. Psychol. 84, $445-452$.

Wimersma Greidanus, T.B., 1982. Disturbed behavior and memory of the Brattleboro rat. Ann. N.Y. Acad. Sci. 394, 655-662.

Wimersma Greidanus, T.B., van Ree, J.M., de Wied, D., 1983. Vasopressin and memory. Pharmacol. Ther. 20, 437-458. 